\title{
Classification of Spam Text using SVM
}

\author{
Gaddam Akhil Reddy ${ }^{1}$ \\ Department of Information Technology \\ Sreenidhi Institute of Science \& Technology, Hyderabad, Telangana, India \\ Email-akhilreddyg21pa@gmail.com \\ Dr. B. Indira Reddy ${ }^{2}$ \\ Professor, Department of Information Technology \\ Sreenidhi Institute of Science \& Technology, Hyderabad, Telangana, India \\ Email-bindira@sreenidhi.edu.in
}

\begin{abstract}
The necessity for spam detection is particularly pertinent nowadays, as there is no quality control over social media, and users have the ability to distribute unverified material, therefore facilitating fraud and deceit. Spam detection can aid in the prevention of such fraud. This scenario has developed mostly as a result of the distribution of disparate, unconfirmed information via shopping websites, emails, and text messages (SMS). There are several ways of categorising and identifying spam. Each of them has certain advantages and disadvantages. The machine learning model "Support Vector Machine" is employed to detect spam in this case. SVM is a basic concept: the method proposes a line or hyperplane to classify the data. The model can categorise any type of text into a given category after being fed a set of labelled training data for each category.
\end{abstract}

Keywords: SVM, Naive Bayes, KNN, Under Sampling, Vectorization, TF-IDF.

\section{INTRODUCTION}

Black and white lists, keyword matching, and header information analysis were common spam filtering approaches in the past. In today's Internet landscape, however, the aforementioned techniques are less useful. Today, the increase in the widespread use of the internet means it is more difficult to develop a spam filter that distinguishes spam messages from messages that are essential and imperative. Statistical learning techniques are being used to build a machine learning-based solution to solve this problem. The most pressing issue at present is building a spam filter capable of quickly and effectively catching all of the spam.

Suspicious and dishonest people seek out chat services such as Google Chat, AOL Chat, and Facebook Chat, and so are known as spammers. A handful of social networking systems provide a client register that also stores statistical data, such as date of birth, age, and gender. Marketers can access this data without the user's awareness and then bombard the user with ads for unrelated goods, services, or concepts. It is a particularly effective channel for spammers because it is rarely hampered by firewalls. Primarily, this is intended for those people who visit to find new companions on the internet, who then inadvertently install dangerous software on their devices. This kind of spam concentrates on instructional information on how to take care of a mobile phone. If the service aims to provide a certain service that is free for the user's mobile device and that comes with a recurring fee, then this might be very frustrating for the user. This kind of communication often consists of a few marketing plans and sales campaigns for distinct items. Service providers can employ this approach in some instances to encourage customers to use their services.

Bayes, SVM, and KNN are the most often utilized methods for message categorization in the current environment. Categorizing texts according to their subject is a widely used filtering technique. On the other hand, it does have certain drawbacks. While it appears to be negative, the truth is that self-contained words are seen to be less unique. Although most of the time, this assumption doesn't hold, in some cases it is completely accurate. Because of this, there is a limit to the growth in categorization accuracy. With regards to KNN, the quality of the data affects the accuracy. If the data is of low quality, then this might lead to conflict. Additionally, it is computationally costly because of the magnitude of the data and its complexity, as well as being sensitive to data volume and irrelevant 
characteristics. The most effective classification strategy for text analysis is the use of the SVM, a statistical classification method that focuses on two classification categories. It is well-known because of its exceptionally high classification accuracy that SVM is often used for text categorization.

There are five categories that this work is split into, as shown below. The first portion of this chapter offers a summary of the study's findings. This section includes a brief description of previous initiatives and their respective research aims. In this study, the research approach described in section three was used. In section four, the findings of the research are discussed. A summary of the research and what's next is included in the concluding section.

\section{LITERATURE SURVEY}

When investigating spam detection, Hidalgo ran an experiment using four different algorithms, such as Naive Bayes, C4.5, PART, and Support Vector Machine (SVM). SVM, with a 90\% accuracy, was discovered to be the most accurate algorithm for finding spam text messages. Another 2013 research investigated several classifier methods for spam text message filtering. The three methods boosted are Naive Bayes, Logistic Regression, and Boosted C4.5. The number of text messages in this research totals 1,324, including 1,002 text messages that are ham messages and 322 that are spam. English SMS texts are used here.

In 2011, an experiment was conducted to see if there was still SMS spam on a mobile phone user's device. This study employed the Naive Bayes technique to classify the text. Once the data has been collected, filtered, and trained, it is immediately accessible on the cell phone. This study found that the models produced in the next stage were $90.64 \%$ accurate in identifying spam text messages. In the same year, Lee and Hsieh researched the usage of CAPTCHA and a secret sharing methodology as an SMS confirmation method. They examined SMS spam filtering as well. They stated that the authentication technique was so simple that very little processing was required. The two major features of an identity verification service are identity verification and user participation verification. Additionally, this method works well in a mobile context.

Support Vector Machine is the finest spam classification algorithm, claims Delany. Fernandes' recent work is also consistent with earlier research. In this research, Optimum-Path Forest (OPF) is employed to analyze spam text message identification, which is then compared to SVM. This research reveals that SVM outperforms other machine learning methods, with an accuracy of 97.79 percent. OPF obtained $92.23 \%$ accuracy.

\section{Proposed Algorithm}

\subsection{Text Messages Collection}

For the most part, messages are composed of text and multimedia elements. Communication via text messages is more common in daily life than previously believed. The primary goal of this research is to investigate the classification of text messages, which includes carriage returns, line feeds, and spaces. This refers to the person who receives the message, the message's content, the person who sent the message, and the time the message was delivered. The objective of this research is to analyze two different types of text messages: spam and ham. The dataset we will use is called "spam.tsv", and it has 5572 emails in it, of which 747 are spam and 4825 are ham. In this dataset, the target variable is' label ', and everything that deviates is mapped to' ham'. When thinking about the target variable, one may say that it represents what you are predicting or the predictor variable. Classifiers, whether supervised or unsupervised, require the correct target variable so that they can be able to categorize the correct input variable.

$\mathrm{df}=$ pd.read_csv('spam.tsv', sep='tt') 


\section{Architecture:}

\section{Step 1: Data preprocessing}

The extracted information is saved in the relevant database when the material is received. A feature vector was created for each message, with a total of 21700 attributes. The attribute $n$ was either set to 1 if the appropriate word existed in a message or else was assigned to 0 if the term was not present.

Step 2: Description of the retrieved feature

Spam and ham content is removed by the feature extraction modules, after which feature dictionaries and feature vectors are created for the algorithm's input. Feature extraction is utilized to assist in classifier training and verification. In terms of the training component, this module takes note of the number of words that occur in the message text. We use the terms that appear the most frequently as well as terms from the class's core vocabulary. Training messages are represented by feature vectors, as well.

Step 3: Classification of spam

For training, we use standard classification message documents which we preprocess and extract useful information. We save the extracted information as text documents in a prescribed format, which are then broken down into words, after which we extract the spam document's feature vector. Using the feature vector of spam messages, the SVM technique is found to be the most optimal classification method.

Step 4: Evaluation of performance

To measure the efficacy of the above method, Spam Filtering researchers have utilized the most frequently used evaluation tools. Spam Precision (SP), spam recall (SR), and Accuracy (A) are the performance metrics. 


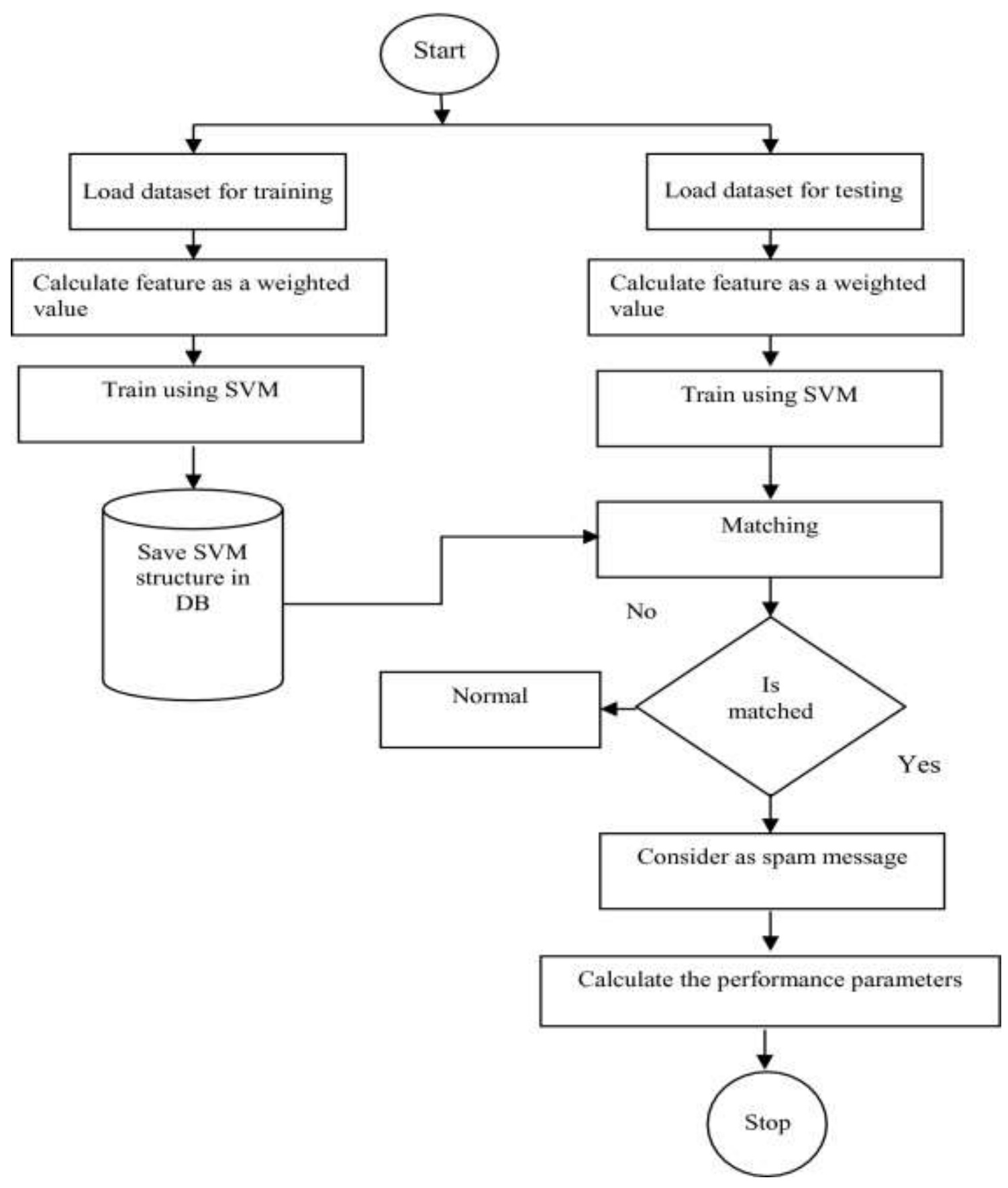

Figure 1. Architecture

\subsection{Purification of Data}

Because the survey has been made available to the public, the raw data collected from the survey can not be guaranteed to be acceptable for use in such a study. As a consequence, the data needs to be cleaned before further processing. Data cleaning is a procedure that finds inaccurate, incomplete, or infeasible data, such as duplicated, inconsistent data, documentation mistakes, or unqualified data, and then fixes or removes them to improve the quality of the data. Previous research required observing the whole collection of data to determine errors and lack of data. 


\subsection{Text Pre-processing}

These text messages should be prepared before any are used during training or testing. Many processing steps, including case folding, punctuation removal, tokenization, URL handling, and stemming, are applied to the text before being used. This study doesn't take MWEs (Multiword Expressions) or text relevancy into consideration. While MWE may play a role in document clustering, the brief text currently lacks the relevance that MWE may have. For the sake of this explanation, we'll start by assuming that the text message is already all lower-case. To verify there are no case-sensitive problems, perform this step. A second factor is that the text message is stripped of any punctuation. It is assumed that no punctuation has an impact on the final result. Thirdly, tokenization is required because data training and testing techniques establish each token's probability in the text messages. Other messages you may get contain a URL or phone number, especially those with offers of money in exchange for fraud. Despite similar text messages, these URLs or phone numbers are distinct. Due to this, the findings in this study regard multiple URLs or phone numbers as interchangeable resources. We have replaced all URLs and phone numbers with the terms "URL address" and "phone number." After text pre-processing, the last stage is known as stemming. To retrieve the base word of the token, perform the following steps.

\subsection{Data set balancing (Under-sampling or Down Sampling)}

To offset the increased data, the abundant class size is reduced. To obtain a more equitable distribution of classes, it limits the number of majority samples. It may exclude valuable information, as it involves removing observations from the original data set. While it helps with the resolution of memory problems.

ham.sample $=$ ham.sample $($ spam.shape[0])

ham.shape,spam.shape

$((747,4),(747,4))$

This data will be loaded into a new dataset.

OData $=$ ham. append $($ spam, ignore index $=$ True $)$

\subsection{Text Vectorization}

We found that the Bag of Words Model, the word vectorizer, was impractical for our Spam or Ham classifier after the examination because it only contains the most basic words. Each vectorizer has its own unique set of advantages, but figuring out which one to use is not always clear. Due to its simplicity of usage and effectiveness in vectorizing documents such as text messages, the TF-IDF vectorizer was utilized in this instance. Vectorization of documents is accomplished by producing a TF-IDF statistic between the document and each phrase in the lexicon. Every statistic in the document vector is applied.

To choose TF-IDF, we will have to choose the granularity of our vectorizer. It is a frequent option to assign each word its term instead of using a tokenizer. White space and special characters are used to split documents into tokens. Tokenizers can retrieve data that other types of analyzers may miss. While tokenizers may struggle with colloquial English, they will have problems with URLs and emails. This approach means we'll be employing a word-level analyzer, which groups words into categories. Before training the vectorizer, we divide our data into two sets: a training set and a testing set. We set aside $30 \%$ of our data for testing.

from sklearn.model_selection import train_test_split

x_train, $x \_$test, y_train, y_test $=$train_test_split

$($ OData['message'], OData['label'], test_size $=0.3$, random_state $=0$, shuffle $=$ True $)$

\# vectorizer training 
from sklearn.feature_extraction.text import TfidfVectorizer

svm = Pipeline ([("tfidf",TfidfVectorizer()) , ("classifier",SVC(C= 100, gamma ='auto'))])

\subsection{Building and Testing the Classifier}

During this step, classifiers will be selected and tested on the training set to see which one performs the best. We could assume that a Support Vector Machine performs well enough to ensure consistency. A higher value of C usually leads to a hyperplane with a smaller margin because it places a larger priority on precision over margin. The purpose is to detect the term $\mathrm{C}$ to influence objective function as a regularisation. The grid search can properly adjust these settings.

from sklearn import svc

svm.fit(X_train, y_train)

\subsection{Performance Metrics:}

The current framework was evaluated using the following standard metrics. To evaluate accuracy, recall, and precision, a specific dataset is needed. We have to figure out how many instances were correctly identified as belonging to the class, i.e., True Positives, the number of instances that were correctly identified but did not belong to the class, i.e., True Negatives, and the instances that were mistakenly classified as class examples, i.e., False Positives, as well as instances that weren't identified as class examples, i.e., False Negatives.

Accuracy may be defined as the total number of correct predictions to the total number of predictions.

Accuracy $=(\mathrm{TP}+\mathrm{TN}) /(\mathrm{TP}+\mathrm{TN}+\mathrm{FP}+\mathrm{FN})$.

Where TP $=$ True Positives, $\mathrm{TN}=$ True Negatives, $\mathrm{FP}=$ False Positives, FN $=$ False Negatives

\section{Recall (Sensitivity)}

A statistical measure known as the recall is used to represent the ratio of successfully grouped positive models to the total number of positive models. The class was acceptable provided the recall rate was high.

Recall $=\mathrm{TP} /(\mathrm{TP}+\mathrm{FN})$

\section{Precision}

To estimate the accuracy of our classifier, we divide the total number of correctly classified examples by the number of predicted positive cases.

Precision $=\mathrm{TP} /(\mathrm{TP}+\mathrm{FP})$.

\section{F-Measure}

It has an estimator that speaks to both measures (Precision and Recall) since we have two metrics (Precision and Recall). We developed an F-measure based on Harmonic Mean rather than Mean since it is more sensitive to unusual features. The formula for computing the F-measure may be found in the table below. The F-Measure will always be closer to the estimated precision or recall value than the expected precision or recall value.

F-measure $=(2 *$ Recall $*$ Precision $) /($ Recall + Precision $)$.

Let's test it. 
from sklearn.metrics import accuracy_score

from sklearn.metrics import confusion_matrix

from sklearn.metrics import classification_report

y_pred $=$ svm.predict $\left(\mathrm{x} \_\right.$test $)$

print(accuracy_score(y_test, y_pred))

$\operatorname{print}($ confusion_matrix(y_test, y_pred))

print(classification_report(y_test, y_pred))

\section{RESULT}

In this study, we used the "spam.tsv" file, which contains 5572 emails, 747 of which are spam and 4825 of which are ham. We achieved an accuracy score of 95.32 percent after applying all the techniques of data collecting, preprocessing, balancing, and then applying the Support vector machine algorithm to categorise the messages into spam or ham.

Accuracy Score:

print(accuracy_score(y_test, y_pred))

0.9532293986636972

Figure 2. Accuracy Score

Confusion Matrix:

print(confusion_matrix(y_test , y_pred))

$\left[\begin{array}{ll}223 & 4\end{array}\right]$

[ 17 205]]

Figure 3. Confusion Matrix

Classification Report:

\begin{tabular}{rrrrr}
\multicolumn{4}{l}{ print(classification_report(y_test, y_pred)) } \\
precision & recall & f1-score & support \\
ham & 0.93 & 0.98 & 0.96 & 227 \\
spam & 0.98 & 0.92 & 0.95 & 222 \\
& & & & \\
accuracy & & & 0.95 & 449 \\
macro avg & 0.96 & 0.95 & 0.95 & 449 \\
weighted avg & 0.95 & 0.95 & 0.95 & 449
\end{tabular}

Figure 4. Classification Report 
Validating our model through the use of real-world examples :

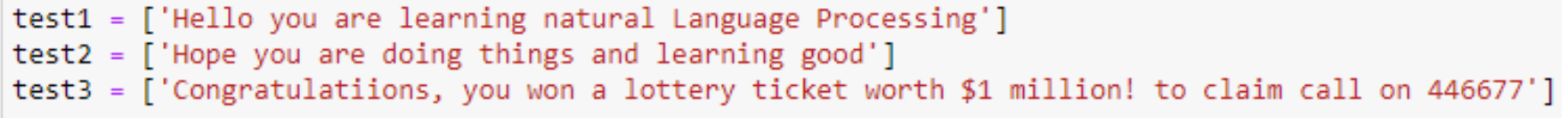

Figure 5. Random Input

\section{CONCLUSION}

Unfortunately, the need for total accuracy in spam classifications has not yet been fully satisfied, according to this research. To put it bluntly, spam is one of the biggest nuisances to the global PC environment. We propose a novel spam detection method in this study that is effective at telling spam apart from its contents. With sparse data structures and suitable recall and precision values, SVM can handle data with a huge number of features. Also, the SVM is often viewed as a critical kernel method, and this is very important in machine learning. The user can either filter spam or continue to receive real emails. During classifying a collection of datasets, the recommended classifier gets a classification accuracy of 95.32 percent. Next, a mobile application will be developed using the model that was built in this study to detect spam text messages. To be able to better identify spam text messages, it is also required to construct a server that will be used to generate a better model. It is necessary to introduce fresh spam messages to the model regularly to improve the model. It may be used as a tool to provide the most current spam text messages, delivering the most recent spam text messages that have been flagged by the user. The resulting server model creates a new form of spam text message detector.

\section{Acknowledgment}

I would like to express my deep gratitude to my professor, Dr. B. Indira Reddy, for guiding me through the process of writing this research paper.

\section{REFERENCES}

[1] D. Puniškis, R. Laurutis, R. Dirmeikis, An

Artificial Neural Nets for Spam e-mail

Recognition, electronics and electrical engineering ISSN 1392 - 1215 2006. Nr. 5(69)

[2] H. Najadat, N. Abdulla, R. Abooraig, and S. Nawasrah, "Mobile SMS spam filtering based on mixing classifiers," Int. J. Adv. Comput. Res., vol. 1, no. 1, pp. 1-7, 2014.

[3] Q. Xu, E. W. Xiang, Q. Yang, J. Du, and J. Zhong, "SMS spam detection using noncontent features,' IEEE Intell. Syst., vol. 27, no. 6, pp. 44-51, Nov./Dec. 2012.

[4] A. Modupe, O. O. Olugbara, and S. O. Ojo, "Filtering of mobile short messaging service communication using latent Dirichlet allocation with social network analysis," in Transactions on Engineering Technologies. Springer, 2014, pp. 671-686. 
[5] A. Skudlark, "Characterizing SMS spam in a large cellular network via mining victim spam reports," in Proc. 20th ITS Biennial Conf., Rio de Janeiro, Brazil Nov./Dec. 2014, pp. 1-23.

[6] C. F. M. Foozy, R. Ahmad, and M. F. Abdollah, "A framework for SMS spam and phishing detection in malay language: A case study," Int. Rev. Comput. Softw., vol. 9, no. 7, pp. 1248-1254, 2014.

[7] Youn and Dennis McLeod, “ A Comparative Study for Email Classification, Seongwook Los Angeles", CA 90089, USA, 2006.

[8] L. Chen, Z. Yan, W. Zhang, and R. Kantola, "TruSMS: A trustworthy SMS spam control system based on trust management," Future Generat. Comput. Syst., vol. 49, pp. 77-93, Aug. 2015.

[9] H. Saeed and W. Waheeb, "The performance of soft computing techniques on content-based SMS spam filtering," M.S. thesis, Dept. Elect. Eng., Univ. Tun Hussein Onn Malaysia, Johor, Malaysia, 2015.

[10] E.-S. M. El-Alfy and A. A. AlHasan, "Spam filtering framework for multimodal mobile communication based on dendritic cell algorithm," Future Generat. Comput. Syst., vol. 64, pp. 98-107, Nov. 2016.

[11] E. Ezpeleta, U. Zurutuza, and J. M. G. Hidalgo, "Short messages spam filtering using personality recognition," in Proc. 4th Spanish Conf. Inf. Retr., 2016, p. 7.

[12] M. Z. Rafique and M. Abulaish, "Graph-based learning model for detection of SMS spam on smartphones," in Proc. 8th Int. Wireless Commun. Mobile Comput. Conf. (IWCMC), Aug. 2012, pp. 1046-1051.

[13] T. A. Almeida, T. P. Silva, I. Santos, and J. M. G. Hidalgo, “Text normalization and semantic indexing to enhance instant messaging and SMS spam filtering,"' Knowl.-Based Syst., vol. 108, pp. 25-32, Sep. 2016. 